\title{
Plan estratégico provincial de turismo de Tarma: PEPTUR Tarma 2015-2021
}

\section{Provincial tourism strategic plan Tarma: Tarma PEPTUR 2015-2021}

\author{
Oscar Raúl Rojas Guere' / Andrea Soledad Orihuela Ponciano² \\ 'oscarojasguere@hotmail.com \\ ªndrea.orihuela@hotmail.com \\ Facultad de Ciencias Aplicadas - Tarma, Universidad Nacional del Centro del Perú
}

\section{RESUMEN}

La investigación se realizó con el propósito de formular estrategias más eficientes para lograr el Desarrollo Turístico de la Provincia de Tarma a través del Planteamiento de un Plan Estratégico de Turismo para la Provincia de Tarma, para ese fin pretendimos contestar la siguiente interrogante: ¿̇ué características debe poseer el "Plan Estratégico Provincial de Turismo de Tarma: PEPTUR TARMA 20152021 para consolidar el desarrollo turístico de la provincia?

Se efectuó el proceso de Planificación Estratégica del Turismo a nivel de la Provincia de Tarma, aplicándose una metodología acorde que involucró el análisis de los antecedentes, el marco normativo, el Análisis FODA y la implementación de las estrategias, programas y acciones correspondientes.

Palabras clave: Plan Estratégico de Turismo - Tarma.

\section{ABSTRACT}

The research was conducted in order to develop more effective strategies for achieving the Tourism Development of the Province of Tarma through the approach of a Strategic Plan for Tourism for the Province of Tarma, for that purpose we sought to answer the following question: What characteristics you must have the "Provincial Tourism Strategic Plan Tarma: Tarma PEPTUR 2015-2021 to strengthen tourism development in the province? the process of Strategic Tourism Planning was conducted at the level of the Province of Tarma, applying a consistent methodology involving the analysis of the background, the regulatory framework, SWOT analysis and implementation of strategies, programs and corresponding actions.

Keywords: Strategic Plan Tourism - Tarma. 


\section{INTRODUCCIÓN}

\section{Objetivo:}

Formular el "Plan Estratégico Provincial de Turismo de Tarma: PEPTUR TARMA 2015-2021, aplicando los principios de sostenibilidad y convocando a los diversos actores que tienen injerencia en el sector turístico en la Provincia.

\section{MATERIALES Y MÉTODOS}

\section{Método de Investigación}

Método Científico. Este método fue aplicado desde el inicio de la investigación, asegurando la Método Inductivo. En el desarrollo de nuestra investigación se utilizó el método inductivo lo cual nos permitió hacer el estudio, partiendo de algunos aspectos particulares como la necesidad de planificación del sector turismo, para llegar a nuestros objetivos mediante la generalización de los resultados.

Método Descriptivo. En este estudio empleamos el método descriptivo a partir de la observación que luego se tradujeron en datos utilizables para conocer las situaciones, predominantes en las condiciones del turismo en la provincia de Tarma. Su meta no se limita a la recolección de datos, sino a la predicción e identificación de las relaciones que existen entre dos o más variables.

Talleres de Planificación Estratégica. Donde se consolidó la formulación del diagnóstico y el direccionamiento estratégico; correspondiendo a una estrategia participativa.

\section{RESULTADOS Y DISCUSIÓN}

\section{VOCACIÓN TURÍSTICA}

\section{Tarma: "fe, algarabía, tradición y aventura"}

Se llegó a esta conclusión porque Tarma está caracterizada en el mercado turístico nacional por su Semana Santa, por sus alfombras de flores, su religiosidad entremezclada con la celebración tradicional de sus pueblos durante el mes de mayo, la muliza de sus carnavales y la práctica de actividades turísticas de aventura bajo un imponente cielo azul que los visitantes pueden disfrutar durante su estancia.

\section{DIAGNÓSTICO}

Contexto del desarrollo del turismo en la Región Junín.

De acuerdo con lo señalado en el Plan Estratégico Nacional de Turismo - PENTUR 2012 - 2021, el turismo es una fuerza económica, social y ambiental de proporciones globales. Se trata de la mayor industria del mundo: para el año 2000, el turismo superó a otras industrias en exportaciones totales a nivel mundial, con más 550 mil de millones de dólares.

Desde 1990 hasta el 2003, los turistas internacionales han aumentado en un $40 \%$. En términos generales, el mercado turístico internacional ha seguido durante los últimos años una tendencia de crecimiento tal que, de mantenerse, hará que el mercado se duplique en los próximos dieciocho años hasta llegar a los mil seiscientos millones de viajeros.

El potencial para el desarrollo del turismo en la Región Junín está dado no sólo por sus recursos arqueológicos - culturales, sino también por sus recursos ecológicos; los cuales junto con la oferta extendida hacía la región central que conforman las regiones de Lima, Huánuco, Huancavelica y Ayacucho, deben permitir que dichas regiones en su conjunto y la Región Junín en especial, se conviertan en el segundo centro de atracción del turismo en el Perú, como contraparte al turismo en el sur.

Las características del territorio regional permitiría que el turismo a desarrollar se base en la nueva tendencia de la demandas de viajes, con los denominados TIE (Tours de Interés Especial), con actividades recreativas, de aventura, misticismo, gastronómicas y ecológicas.

PENTUR 2012 - 2021:

Visión al 2021:

El Perú está posicionado en el mercado nacional $y$ en los principales mercados 
emisores internacionales, como un destino turístico seguro, competitivo y confiable, líder en América Latina, que tiene: identidad turística propia; conservación y uso racional de su mega diversidad natural, histórica e intercultural; un marco legal e institucional adecuado y una gestión participativa y descentralizada; una oferta de productos y servicios turísticos de calidad, diversificada y sostenible.

\section{Objetivo General:}

Alcanzar el desarrollo sostenible del turismo en el Perú.

\section{Objetivos Estratégicos:}

1. Desarrollar una oferta turística competitiva y sostenible.

2. Desarrollar una cultura turística que garantice la seguridad del visitante, una visión y valores compartidos e impulse las buenas prácticas en la prestación de los servicios turísticos.

3. Fortalecer las instituciones vinculadas a la actividad turística.

4. Incrementar de manera sostenida la demanda del turismo receptivo a fin de generar mayor ingreso de divisas al país, así como el turismo interno a fin de asegurar una redistribución de recursos a nivel nacional.

PERTUR JUNÍN 2004 - 2014.

\section{Visión y misión}

Como producto de los talleres preliminares se obtuvieron diversas propuestas locales de Visión y Misión, las cuales fueron discutidas y consensuadas durante el taller final realizado en La Merced en agosto del año 2003. Producto de este proceso se concluyó en la siguiente Visión y Misión regional 2003-2013:

\section{VISIÓN}

Junín, región líder en turismo sostenible, vivencial, cultural, de aventura y ecoturismo a nivel nacional e internacional, con oferta diversificada en productos turísticos que fortalecen la identidad regional y contribuyen a la conservación de los recursos naturales y culturales, con actitud participativa de la población y autoridades, generando el desarrollo socio-económico de la región.

\section{MISIÓN}

Promover, desarrollar y ofertar al mercado nacional e internacional productos turísticos diversificados y de calidad, enfocados principalmente en la cultura viva y naturaleza, con la participación activa de la población local, empresarios y autoridades.

\section{Turismo en la Provincia de Tarma:}

Figura 1 Contexto de I Turismo 1990 - 2005

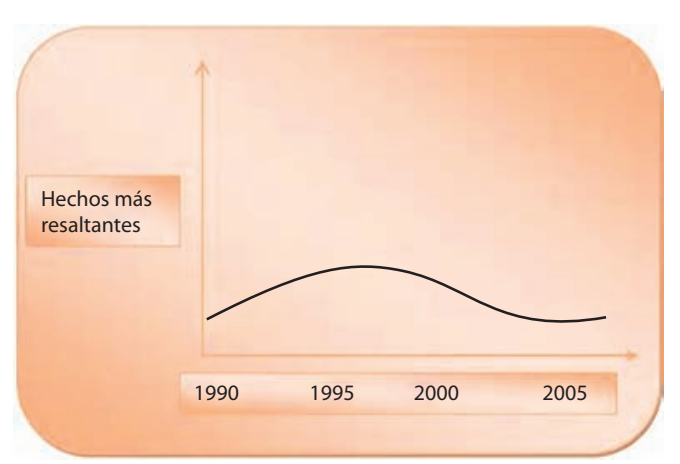

1990: el turismo en Tarma de 1985 a 1990 fue descendiente, debido a los altos índices de violencia política que vivía nuestro país.

1995: para este periodo el turismo en Tarma comenzó a resurgir por la pacificación de la violencia política y el fomento que se dio al turismo.

2000: para este periodo el turismo siguió aumentando como resultado de la inversión en infraestructura que se dio y la poca competencia. 2005: para este periodo el turismo en Tarma se vio afectada por la informalidad de buena parte del sector así como la afluencia de más turistas y la limitada infraestructura turística de calidad para atender, situación que se ve hasta la actualidad acompañada de la escasa cultura de atención de atención de calidad y calidez al turista. 


\section{Determinación de Valores:}

Efectuados los talleres participativos se consensuaron los valores fundamentales para el desarrollo de la actividad turística:

$\checkmark$ Amabilidad

$\checkmark$ Respeto mutuo

$\checkmark$ Puntualidad

$\checkmark$ Lealtad

$\checkmark$ Responsabilidad

$\checkmark$ Honestidad

$\checkmark$ Solidaridad

$\checkmark$ Cooperación

\section{Direccionamiento Estratégico:}

\section{Visión al 2021:}

"Tarma al 2021, es un destino turístico líder y está posicionado en el mercado de turismo interno de la región Junín y la Zona Centro, como destino turístico de fe, tradición, algarabía y naturaleza"

\section{Misión:}

Proporcionar servicios turísticos de calidad, desarrollando las capacidades de los actores de la actividad turística y poniendo en valor los recursos turísticos de los nueve distritos de la Provincia de Tarma.

\section{Objetivos Estratégicos:}

1. Diversificar y consolidar la oferta turística a partir de nuestros recursos turísticos de manera competitiva y sostenible, desarrollando circuitos internos.

2. Desarrollar una cultura turística que se refleje en el buen trato al turista, un entorno rural y urbano limpio, seguro y ordenado.

3. Impulsar la competitividad y sostenibilidad del sector, fortaleciendo los gremios de turismo, así como los gobiernos distritales, comunidades, asociaciones y centros de formación turística.

4. Desarrollar de manera sostenida la demanda del turismo receptivo y nacional a través de un mejor conocimiento del comportamiento y requerimiento de los turistas actuales y potenciales.

\section{ANÁLISIS FODA:}

El siguiente análisis interno y externo del sector turístico en la Provincia de Tarma es producto del trabajo de grupo con actores involucrados desarrollado en el Taller de análisis respectivo:

\section{Análisis interno}

Se efectuaron talleres con los empresarios de turismo y los principales actores de la actividad turística y se identificaron los principales recursos turísticos de la provincia de Tarma.

\section{Fortalezas:}

$\checkmark$ Existencia de normas nacionales e internacionales de protección ambiental.

$\checkmark$ Existencia del proceso de descentralización del país.

$\checkmark$ Diversidad de zonas climáticas, con abundantes recursos naturales.

$\checkmark$ Población dispuesta a ser organizada (comités de defensa ambiental).

$\checkmark$ Recursos humanos con capacidad profesional y compromiso con el desarrollo local.

$\checkmark$ Existencia de instituciones públicas y privadas involucradas y comprometidas con el desarrollo del turismo local.

$\checkmark$ Existencia de un vasto patrimonio cultural arqueológico.

$\checkmark$ Existencia de un importante número de centros de estudios superiores y tecnológicos.

$\checkmark$ Existencia de gastronomía provincial de reconocimiento nacional.

$\checkmark$ Existencia de recursos locales, nacionales e internacionales para proyectos de desarrollo local y del turismo.

$\checkmark$ Existencia de asociaciones civiles de turismo de la ciudad de Tarma.

$\checkmark$ Existencia de un número importante de profesionales egresados de Hotelería y Turismo.

$\checkmark$ Existencia de actividad productiva de artesanías.

$\checkmark$ Área Natural protegida con potencial ecoturístico. 


\section{Debilidades:}

$\checkmark$ Crecimiento urbano desordenado, falta de catastro.

$\checkmark$ Deficiente presupuesto.

$\checkmark$ Poco liderazgo en Turismo.

$\checkmark$ Incipiente planificación del desarrollo local en turismo.

$\checkmark$ Inexistencia de rellenos sanitarios en circuitos naturales.

$\checkmark$ Poca educación ambiental.

$\checkmark$ Poca de decisión política para invertir en Turismo.

$\checkmark$ Carencia de programas y proyectos culturales.

$\checkmark$ Poca identidad y conocimiento de los valores de la cultura y patrimonio local.

$\checkmark$ Inadecuado uso de los recursos.

$\checkmark$ Inadecuado currículo educativo.

$\checkmark$ Carencia de políticas de transferencia tecnológica.

$\checkmark$ Escasa promoción para desarrollar actividades económicas vinculadas al turismo.

$\checkmark$ Líneas de transporte turístico local inexistentes.

$\checkmark$ No hay una adecuada infraestructura de apoyo al servicio turístico.

$\checkmark$ Inventario turístico no actualizado.

$\checkmark \quad$ Mínimo desarrollo tecnológico.

$\checkmark$ Escasa información para la toma de decisiones.

$\checkmark$ Falta de conocimiento de la normatividad turística.

$\checkmark$ Corrupción de las autoridades.

$\checkmark$ Poco fortalecimiento institucional de gremios en turismo

$\checkmark$ Débil planeamiento turístico, inexistencia de plan estratégico.

$\checkmark$ Excesiva burocracia Institucional.

$\checkmark$ Poca Concertación entre las autoridades, la sociedad civil y el sector privado para el planeamiento del desarrollo turístico.

$\checkmark$ Inadecuada infraestructura turística.

$\checkmark$ Poca inversión en turismo.

$\checkmark$ Poca promoción turística.

$\checkmark$ Débil identidad

$\checkmark$ Existencia de muchas barreras para abrir negocios de turismo.

$\checkmark$ Desconocimiento sobre protección y conservación de recursos turísticos

$\checkmark$ Deficiente capacitación en turismo. $\checkmark$ Poca investigación sobre turismo y sus beneficios.

$\checkmark$ Escasa inversión en proyectos de investigación arqueológica.

\section{Análisis Externo:}

\section{Oportunidades:}

$\checkmark$ Inclusión en la agenda regional y nacional al desarrollo ambiental y turístico.

$\checkmark$ Financiamiento externo para la conservación del medio ambiente.

$\checkmark$ Creciente promoción gubernamental para el turismo externa e interna.

$\checkmark$ Creación de instituciones de desarrollo turístico.

$\checkmark$ Afluencia de recursos de cooperación internacional.

$\checkmark$ Biodiversidad y áreas geográficas para desarrollar el turismo.

$\checkmark$ Naturaleza y clima adecuado.

$\checkmark$ Voluntad política para apoyo del turismo.

$\checkmark$ Buena imagen externa del Perú.

$\checkmark$ Desarrollo turístico en regiones vecinas.

$\checkmark$ Proyectos de inversión de países cooperantes.

\section{Amenazas:}

$\checkmark$ Inadecuada administración de recursos no renovables.

$\checkmark$ Invasión y depredación de terrenos agrícolas.

$\checkmark$ Reservas naturales depredadas e invadidas.

$\checkmark$ Alineación cultural.

$\checkmark$ Leyes inadecuadas.

$\checkmark$ Crisis económica mundial.

$\checkmark$ Contaminación ambiental.

$\checkmark$ Poco apoyo de autoridades comprometidas.

$\checkmark$ Poca concertación de los gremios organizados.

$\checkmark$ Orientación de recursos a otras necesidades no prioritarias.

$\checkmark$ Recursos naturales en proceso de extinción.

$\checkmark$ Deterioro de atractivos turísticos.

$\checkmark$ Poca inversión privada.

$\checkmark$ Pérdida de identidad cultural.

$\checkmark$ Desechos a lo largo de las vías que conducen a lugares turísticos.

$\checkmark$ Reducción del turismo por poca seguridad. 


\section{DISCUSIÓN}

Durante Diseño de Plan Estratégico Provincial de Turismo PEPTUR Tarma 2015 - 2021, se analizaron variables cualitativas, sin embargo también se recabó información cuantitativa sobre la cantidad de servicios básicos y el equipamiento turístico.

Los destinos turísticos más evolucionados son aquellos que invirtieron en Planes Estratégicos, sin embargo no basta con la existencia de esta herramienta básica, sino también es imprescindible, la voluntad política y el esfuerzo del sector privado para su implementación exitosa.

La provincia de Tarma, cuenta con diversas herramientas de gestión municipal a partir de las cuales se planifica el desarrollo sectorial de cada sector privado, el sector turismo por su naturaleza integradora y multifacética, implica la interrelación y concatenación de objetivos que necesariamente comprometen a diversos estratos sociales y a la red empresarial de todos los sectores.

Queda como un tema importante de discusión la consecución del mejoramiento de la calidad de servicios turísticos a partir de la Planificación Turística, se presenta como un desafío la aceptación de nuestra propuesta de Plan Estratégico Provincial de Turismo PEPTUR Tarma 2015 - 2021.

\section{REFERENCIAS BIBLIOGRÁFICAS}

1. Instituto Nacional de Estadística e Informática (INEI). (2004). Diagnóstico Socioeconómico de Junín", Huancayo.

2. Huayón Dall Orto, J. (2000). Perú Turismo en el Nuevo Milenio, Proyecto Nacional II" Pag. 125 2da Edición Imp. Talleres Gráficos Avedecor World Perú S.A. Lima.

3. CARE PERÚ. (2004). "Ley Orgánica de Municipalidades". Edit. Gama Gráfica SRL. Lima.

4. Plan Cooperación Perú - UNESCO Programa de las Naciones Unidas para la Educación, la Ciencia y la Cultura.

5. Organización Mundial del Turismo. (1993).
Desarrollo Turístico Sostenible - Guía para Planificadores", Madrid.

6. Schlesinger, Heskett. (1991). The Service Profit Chain. The Free Press, New York,

7. Goleman, D. (1998). What Makes a Leader?. Harvard Business Review.

8. Ministerio de Trabajo y Promoción del Empleo, PROMPYME. (2004). Manual de Mejoramiento Operativo y Comercial en Establecimientos de Hospedaje de Dos Estrellas. Lima. Perú.

9. Stoner, J. y Freeman, R. (1999). "Introducción a la Administración" Editorial Mc Graw Hiil. México.

10. Domingo Begazo, J. (2002). "Megatendencias de Turismo en el Tercer Milenio": Primera Edición, Edit. San Marcos, Lima - Perú.

11. Plan Integral Desarrollo Estratégico y Participativo del distrito de Tarma.

12. Plan de Desarrollo Económico Local de la Provincia de Tarma al 2021. 
Talleres para Formulación del Plan Estratégico Provincial de Turismo PEPTUR Tarma 2015 - 2021
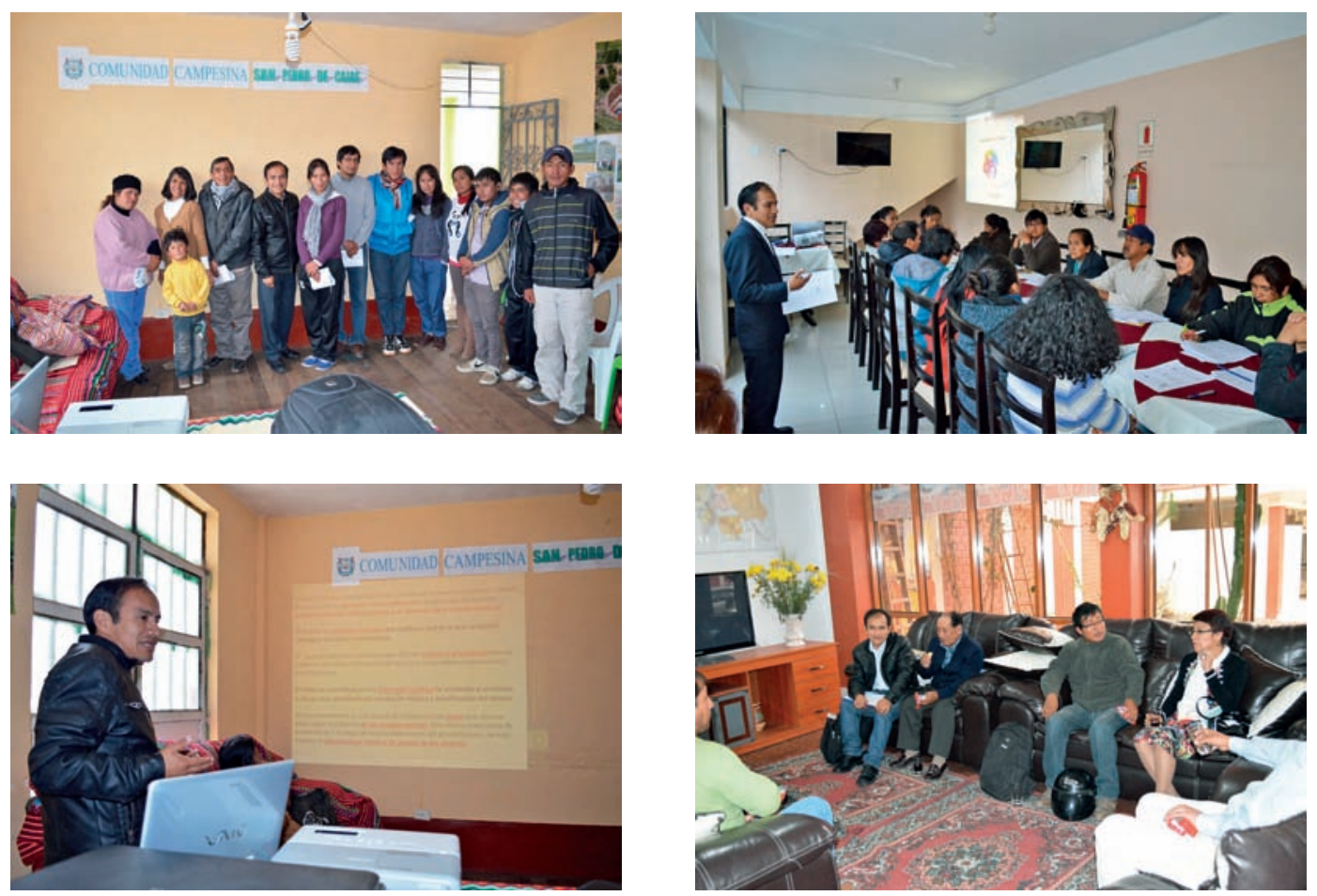